\title{
Elementos motivadores incorporados en los Polimedias plus, vídeos didácticos y screencast para aumentar y mejorar el aprendizaje del estudiante
}

\author{
José Gabriel Ríos Lizanaa, Javier Oliver Villarroya ${ }^{\mathrm{b}}$ y Pilar Aurora Cáceres \\ González ${ }^{\mathrm{c}}$
}

${ }^{\mathrm{a}}$ Técnico de audiovisuales del ICE. jorioli@ice.upv.es, ${ }^{\mathrm{b}}$ Grupo MIST-DSIC. ICE-UPV. fjoliver@dsic.upv.es y ${ }^{\mathrm{C} A s e s o r}$ pedagógico. ICE-UPV.pcaceres@ice.upv.es.

\begin{abstract}
In order to attract students' attention and increase their motivation and participation when they are learning through an audiovisual resource (Polimedia plus, screencast, didactic videos), a system of processing and visualization of generated resources has been implanted that allows the teacher to include a greater amount of material within these resources (reinforcement questions, links to web pages, ...). In this way, it is also achieved that the student is not a mere spectator of the content, he interacts with it to improve his learning.
\end{abstract}

Keywords:Interaction, attention, motivation, Polimedia plus, didactic videos, screencast, training, online, web.

\footnotetext{
Resumen

Con el fin de captar la atención del estudiante e incrementar su motivación y participación cuando aprende a través de un recurso audiovisual (polimedia plus, screencast, vídeos didácticos), se ha implantado un sistema de procesamiento y visionado de los recursos generados que permite al profesor incluir una mayor cantidad de material (preguntas de refuerzo, "links" a páginas web...) dentro de estos recursos. De esta manera, además se consigue que el estudiante, no sea un mero espectador del contenido que está visualizando sino que interactúe con él para que consiga un mayor aprendizaje.
}

Palabras clave: interacción, atención, motivación, Polimedia plus, videos didácticos, screencast, formación, online, web. 


\section{Introducción}

En los últimos años ha habido una proliferación en la elaboración de materiales multimedia de apoyo a la docencia. El mayor hándicap con el que nos encontramos es la escasa interacción de estos materiales, convirtiéndolos únicamente en un medio más de transmisión de contenidos, sin posibilidad de "participación" por parte de los estudiantes, con lo que, en muchos casos, el alumno no ve la totalidad del recurso puesto que no conseguimos mantener su atención hasta el final.

Para intentar paliar estas deficiencias, en el Gabinete de Recursos Educativos y Multimedia (GREM), del Instituto de Ciencias de la Educación (ICE) de la Universitat Politècnica de València (UPV), se está implantando un sistema de software desarrollado íntegramente por José Gabriel Ríos Lizana (técnico de audiovisuales del ICE), que controla todo el proceso de producción de materiales, desde las solicitudes de servicios de los usuarios, pasando por la conversión e incorporación automática de los archivos generados, hasta la publicación en un repositorio web de dichos materiales con la novedad de poder añadir a los mismos, unas capas de superposiciones textuales o gráficas (SDK) en el tiempo y otra de control de la reproducción (EDL), que permite la interactividad con menús y botones que ayudan al profesor a planificar cómo tiene que seguir el estudiante la explicación que se reproduce en estos.

Existen otras aplicaciones web, como EDpuzzle, que también permiten de manera similar generar preguntas en un determinado punto del vídeo. EDpuzzle es una herramienta pensada para que el profesor pueda realizar un mejor seguimiento y evaluación del contenido, más que para el estudiante, ya que le obliga a registrarse e identificarse en la aplicación y acceder con un código que tiene que suministrarle el profesor, no existiendo feedback de las respuestas contestadas. A diferencia de EDpuzzle, esta herramienta que aquí presentamos no obliga al estudiante a identificarse, ni a que el vídeo esté en un repositorio externo (Youtube, khanacademy, learnzillion...) y no controlado por la entidad (el GREM del ICE en este caso). Permite añadir superposiciones textuales o gráficas en el tiempo superpuestas al vídeo, con enlaces a páginas web para que el estudiante tenga más información sobre los temas tratados en el vídeo y permite al profesor una mayor amortización o reutilización de los materiales. Además, mediante la capa de control de la reproducción, permite incluir menús y botones que llevan al vídeo a los puntos correspondientes para que el profesor pueda ofrecer un feedback al alumno sobre la respuesta correcta o errónea de forma inmediata y desde el mismo vídeo que se está reproduciendo, puesto que "salta" a las partes del video donde se encuentra la explicación correspondiente a cada respuesta.

\section{Objetivos}

- Posibilitar la interactuación del estudiante con el recurso educativo durante la explicación del contenido por parte del profesor. 
- Retomar la explicación de forma fácil al poder saltar directamente a los bloques o temas del vídeo definidos por el profesor.

- Posibilitar una mayor cantidad de información al poder añadir enlaces a páginas web y a otros vídeos a un solo click de ratón.

- Conseguir una mayor motivación y atención del alumno al solicitar de manera expresa su interactuación con el vídeo.

- Rentabilizar al máximo una grabación de vídeo al aumentar la reutilización del mismo mediante la inserción de material sensible a cambios en la capa de superposición que puede ser modificada por parte del profesor.

- Potenciar el sistema técnico de producción del GREM para la generación de vídeos.

\section{Desarrollo de la innovación}

Los cambios acontecidos en la Universidad durante los últimos años: integración al Espacio Europeo de Educación Superior, la implantación del Plan Docencia en Red, el desarrollo de la metodología "Clase Inversa", entre otras muchas acciones, han llevado al profesorado a la necesidad de generar y producir un sinfín de materiales didácticos adaptados a las nuevas características del alumnado (generación Z) para conseguir una mayor implicación en su aprendizaje.

En estos cambios, el Gabinete de Recursos Educativos y Multimedia (GREM) de la UPV ha tenido un papel relevante, no sólo en su responsabilidad para facilitar ayuda técnica a los profesores y el apoyo pedagógico ofrecido correspondiente, al formar parte del Instituto de Ciencias de la Educación (ICE) de nuestra universidad, sino también a la hora de plantear innovaciones para mejorar la calidad de los materiales producidos.

En esta línea de innovación, en el GREM se han ido produciendo avances en los últimos años: publicación de los materiales en alta definición y calidad; mejora del plató y de la cabina de edición..., siendo el último de dichos avances el que se presenta en esta comunicación. La idea principal que se propone es no cambiar el sistema técnico de producción de los vídeos, sean estos del tipo que sean, sino que en una revisión posterior se seleccionen los bloques de la explicación y se decida dónde estarán los saltos, las pausas para las preguntas y las diferentes opciones o respuestas, todo ello configurado en una capa de datos de control y asociada al vídeo a la hora de reproducir el mismo.

De esta forma el estudiante ya no visualiza los vídeos de una forma pasiva, sino de una forma activa (como se puede apreciar en la imagen de la Figura 1). 


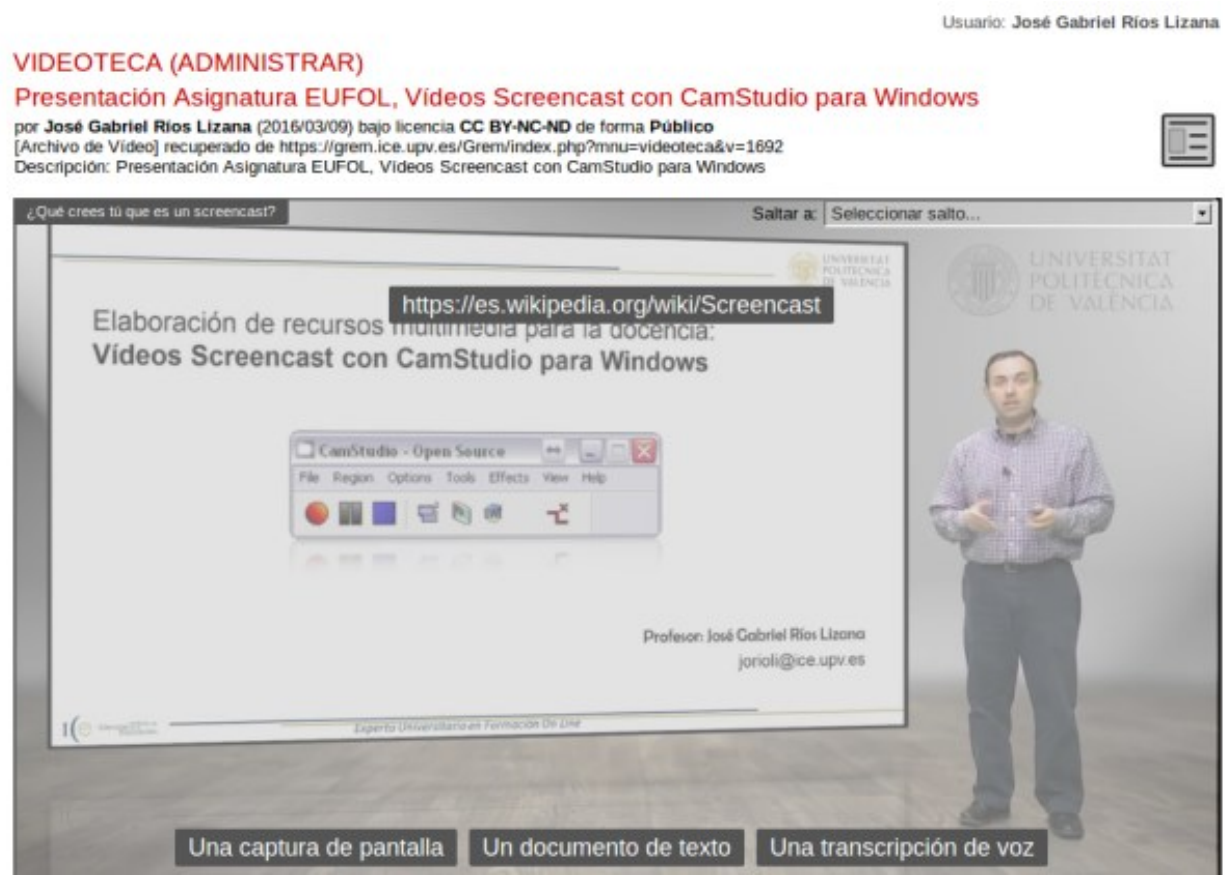

Fig. 1 Imagen del resultado visual de una pregunta con tres opciones posibles y el apoyo de un enlace a una página web de consulta.

La aplicación, como vemos en la imagen de la Figura 2, permite asignar varias opciones, donde el profesor, por ejemplo, tras formular una pregunta al estudiante, lo puede dirigir, en caso de que no sepa contestar dicha pregunta, al tema donde este concepto se explica, bien en un documento almacenado en el repositorio de Poliformat o a una página web, mediante su enlace URL correspondiente; también puede volver al punto del vídeo donde se explica el contenido (es decir, el estudiante puede volver a ver la explicación si no sabe contestar una pregunta determinada).

Por otro lado, incorpora un editor de subtítulos para diferentes idiomas, que pueden seleccionarse, indistintamente, para mostrarse durante la visualización del vídeo.

También permite cinco capas de superposiciones textuales y gráficas que pueden coincidir en el tiempo para el material más sensible a cambios (URL a páginas web, citas de leyes, cursos, fechas...), con funciones de enlaces web a otras páginas o vídeos que permiten aumentar la cantidad de información suministrada al estudiante, así como reutilizar al máximo un vídeo a lo largo del tiempo, ya que esta información se puede cambiar sin necesidad de volver a grabar el vídeo de nuevo. 


\section{VIDEOTECA (ADMINISTRAR)}

Presentación Asignatura EUFOL, Videos Screencast con CamStudio para Windows por Jose Gabriel Rios Lizana (2016/03/09) bajo licencia CC BY-NC-NO de forma Püblico

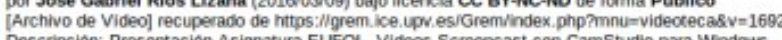
Descripcion: Presentacion Asignatura EUFOL. Videos Screencast con CamStudio para Windows

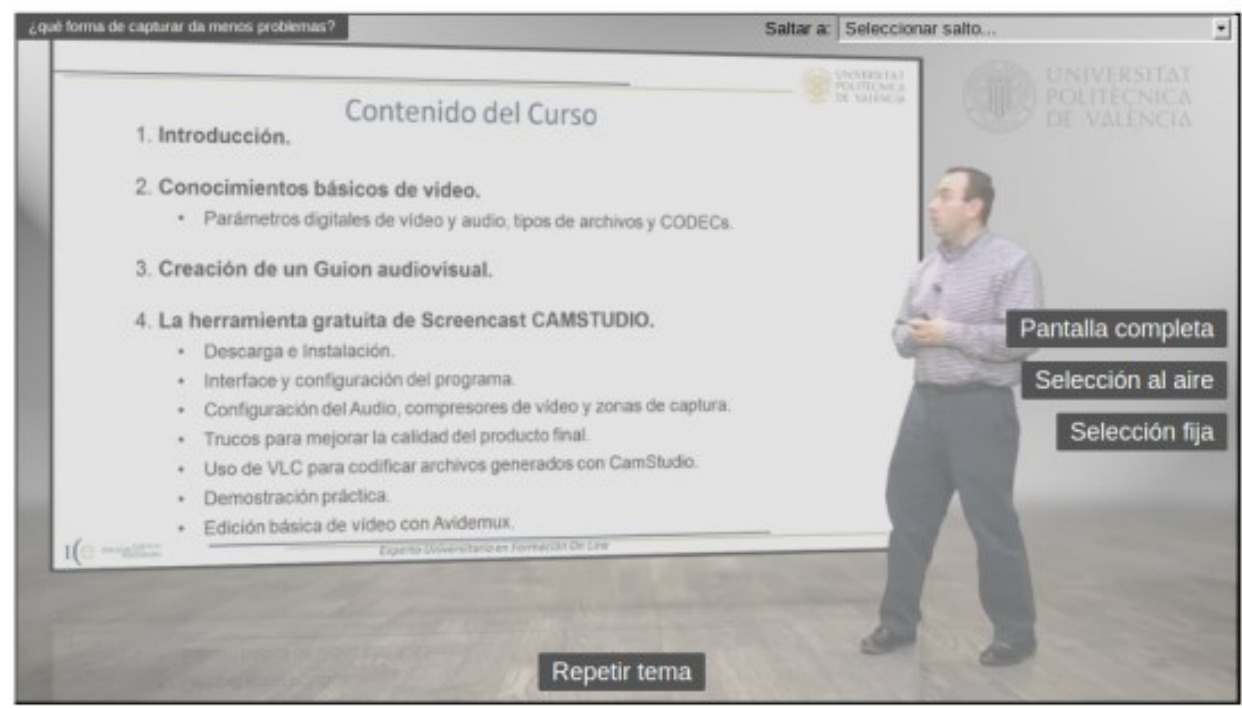

Fig. 2 Imagen del resultado visual de una pregunta con cuatro opciones, siendo una de ellas el volver a visualizar el contenido si el estudiante no sabe la respuesta.

Con la incorporación de todos estos elementos a los videos producidos, estamos seguros que se da un paso muy importante para contribuir a la mejora del aprendizaje de los alumnos.

\subsection{Procesos para la elaboración}

\subsubsection{Producción del vídeo}

La grabación del vídeo se realiza mediante el sistema normal habitual de producción de un Polimedia Plus, vídeo didáctico o screencast, es decir, de forma lineal y en un único archivo de vídeo.

Sin embargo, ahora es necesario que el profesor planifique la explicación de forma que, a la hora de grabar el vídeo:

a) Formule preguntas concretas que tendrán que responder sus estudiantes.

b) Realice pausas, para dar tiempo al estudiante a reflexionar sobre una explicación puntual, buscar una información adicional...

c) Plantee opciones de respuesta para que el estudiante seleccione la que considere correcta.

d) Felicite al estudiante cuando la respuesta ofrecida sea la correcta.

e) Ofrezca feedback al estudiante cuando la respuesta seleccionada sea incorrecta, explicándole, por ejemplo, mediante un contraejemplo por qué es errónea antes de redirigir el vídeo de nuevo a la posición de la pregunta o a otra parte. 


\subsubsection{Publicación del vídeo}

Una vez que el vídeo está grabado, el sistema realiza la conversión y subida del mismo a la plataforma haciendo las correspondientes copias de seguridad y, una vez disponible, se publica automáticamente en el repositorio web, donde se le asigna una dirección URL y queda a disposición del profesor y del resto de la comunidad universitaria para su visualización y posible incrustación en Poliformat u otra plataforma.

Presentación Asignatura EUFOL, Vídeos Screencast con CamStudio para Windows por José Gabriel Ríos Lizana (2016/03/09) bajo licencia CC BY-NC-ND de forma Público Archivo de Video] recuperado de https://grem.ice.upv.es/Grem/index.php?mnu=videoteca\&v=1692 Descripción: Presentación Asignatura EUFOL, Videos Screencast con CamStudio para Windows

Fig. 3 Imagen de la cita que aparece en la parte superior del vídeo.

Como se ve en la Figura 3, el vídeo siempre lleva asociados los metadatos que hacen referencia al mismo: datos de título, autores, fecha de creación, licencia de uso, tipo de archivo y dirección URL, agradecimientos y descripción. Estos datos son visibles siempre incluso cuando se incrusta en otra web, lo que facilita su búsqueda e identificación.

\subsubsection{Generación de clips o partes del vídeo}

\begin{tabular}{|c|c|c|c|c|c|c|}
\hline Saltos y bloques $\vee$ & Edición & $0 \mathbb{B}$ & 00:00:00.000 & 00:00:04.126 & $\therefore \cdots$ & $\checkmark \mathbf{A} \bullet$ \\
\hline - $700 \%$ + & Guardar & A & b) & $\cdots \cdots$ & C [ ] & \\
\hline
\end{tabular}

Fig. 4 Sección del interface de la herramienta de edición, que permite la selección de los tipos de bloques en la pista EDL de la linea de tiempos en un archivo de vídeo grabado de forma lineal.

Una vez se tiene el vídeo publicado, la herramienta ofrece la opción de crear los saltos y bloques (como se puede ver en la Figura 4) que permiten convertir nuestro vídeo en un vídeo interactivo. Esto se realiza de forma gráfica mediante la utilidad de edición proporcionada por la herramienta web, donde de una forma sencilla se pueden marcar y configurar los siguientes tipos de fragmentos o bloques en la pista EDL (Edition Decision List):

- Saltos o bloques: se configura el tiempo de inicio de un tema o el bloque de la explicación y permite asignarle un nombre. Este nombre de bloque se mostrará en la parte superior izquierda mientras se reproduce el video y en la utilidad de “saltar a:". Dicha utilidad aparece en la parte superior derecha de la ventana de vídeo, tanto cuando se pulsa sobre el nombre del tema o bloque actual o cuando el sistema pausa el vídeo para requerir la intervención del estudiante. 


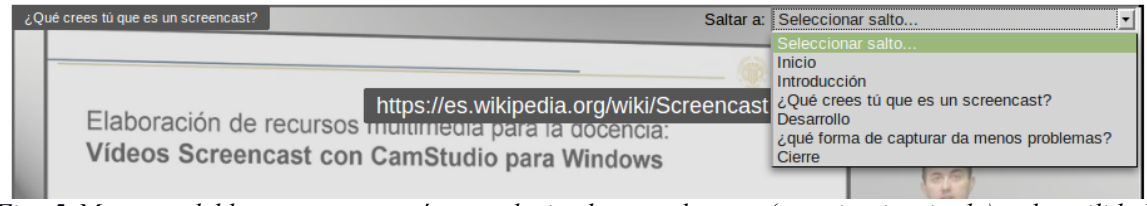

Fig. 5 Muestra el bloque que se está reproduciendo actualmente (superior izquierda) y la utilidad "saltar a" (superior derecha) donde mediante un desplegable se puede seleccionar el punto del temario deseado.

- Saltos o bloques vistos. Permite saltar a determinados puntos del vídeo, pero sólo aparecerán en la utilidad de "Saltar a" cuando el estudiante ya los haya visto, para que pueda repasar, no permitiendo así el avance o salto de temas no visualizados del vídeo.

- Saltos o bloques ocultos. La funcionalidad de este tipo de bloque es permitir saltar a determinados puntos del vídeo, o reproducir determinados bloques del mismo, pero sin que aparezcan reflejados ni en la ventana de vídeo ni en la utilidad de "Saltar a". De esta manera, el estudiante no los ve y se pueden esconder partes del vídeo que el profesor no quiere que visualicen los estudiantes.

- Preguntas. Es un punto, o bloque, donde al final del mismo se pausa el vídeo y se requiere que el estudiante realice alguna acción. Como veremos más adelante, existen distintas tipologías docentes para mantener la atención del estudiante. La pregunta en sí puede ser sobreimpresa mediante texto, pero es mejor que también sea formulada por el profesor en el propio vídeo, y se mostrará en la utilidad "Saltar a". El sistema no tiene límite de cantidad de preguntas a incorporar.

- Preguntas vistas. Al igual que los bloques vistos, las preguntas vistas sólo se mostrarán y se podrán acceder a ellas una vez hayan sido vistas por el estudiante, para que pueda repasar.

- Respuestas. Son bloques del vídeo marcados y asignados a un botón de una pregunta; también pueden contener un enlace a una página web y, por lo tanto, a otros vídeos disponibles online. El sistema permite tener en pantalla hasta 25 botones de respuesta por pregunta, que pueden ser posicionados de forma gráfica, como puede verse en la Figura 6. La ubicación de estos botones en el video es una decisión del profesor en función de lo que más le interese en cada momento. 


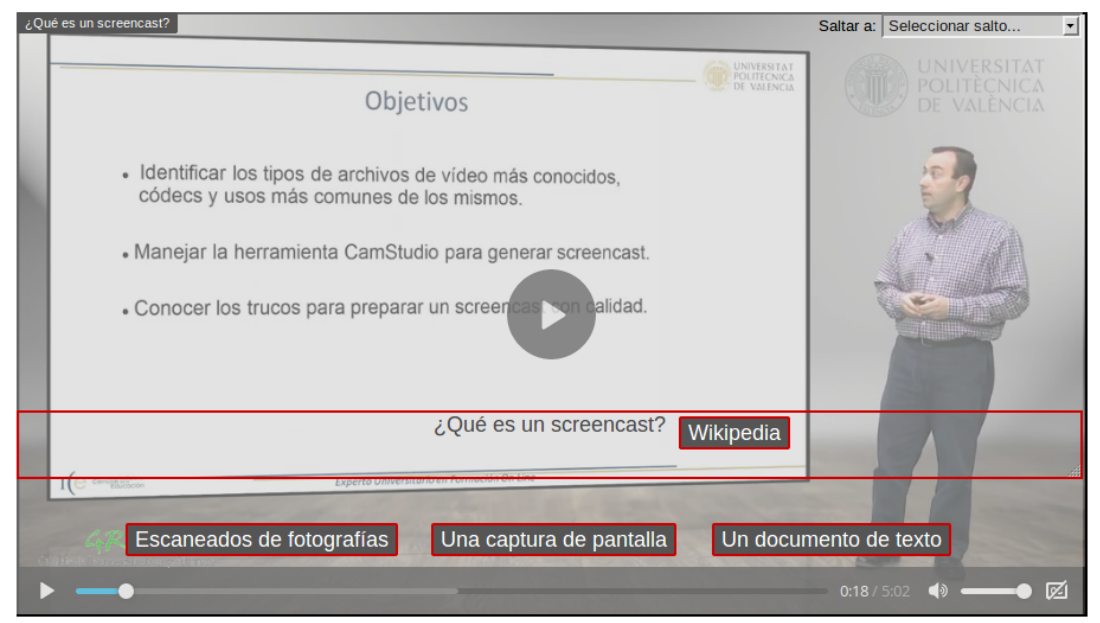

Fig.6. Resalte de los botones y texto de la pregunta para modificar su disposición en la ventana de vídeo mediante la herramienta gráfica moviéndolos con el ratón.

\subsubsection{Generación de contenido superpuesto al vídeo y subtítulos}

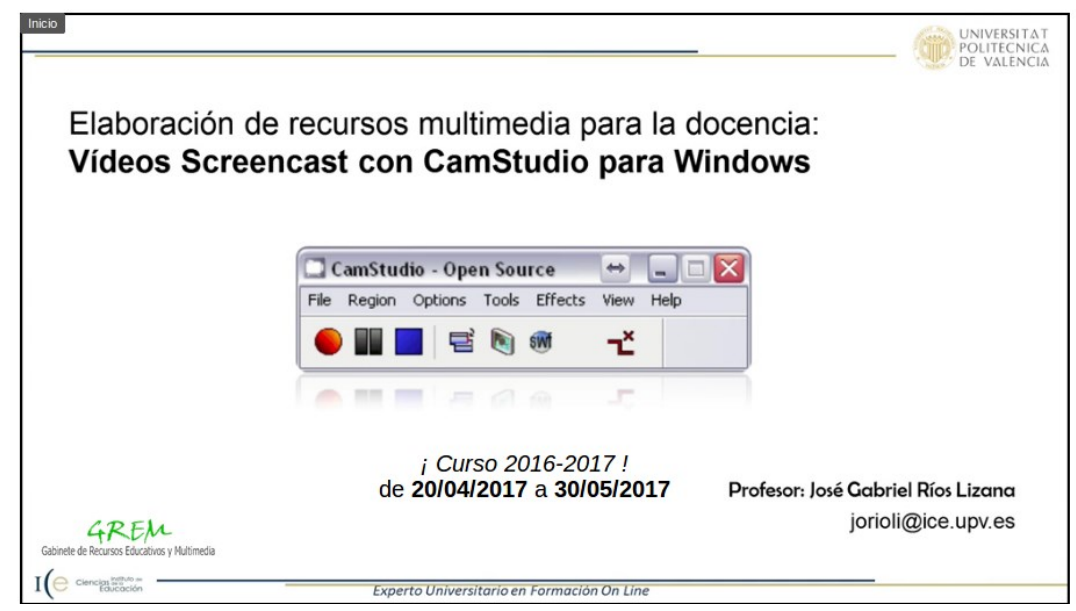

Fig. 7 Visualización de contenido superpuesto al vídeo, como el logotipo del GREM, con un hipervínculo a la página del GREM, y la convocatoria del curso y fechas de inicio y final del mismo.

La herramienta permite la superposición de cinco capas textuales y gráficas simultáneamente en el tiempo que posibilitan tener enlaces a URL, y que se visualizan sobre el vídeo, como se puede ver en la Figura 7, facilitando así personalizar el mismo, añadir información adicional, o amortizar al máximo la utilización del vídeo poniendo en estas capas la información más sensible a cambios en el tiempo, como fechas, páginas web, artículos de leyes, etc. 
También tiene integrado un editor de subtítulos para varios idiomas. Este editor, de forma gráfica y mediante una línea de tiempos permite ir generando los textos y los puntos de entrada y salida para su visualización, un idioma por pista, como se puede ver en la Figura 8 .

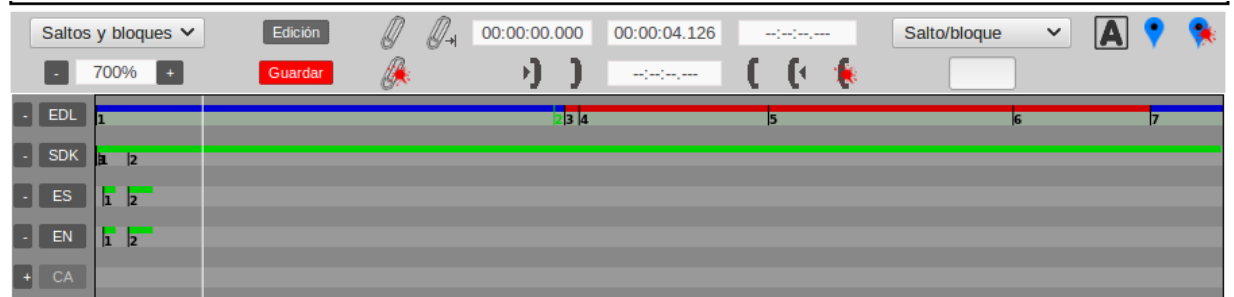

Fig. 8 Visualización de la interface de edición mediante línea de tiempos para las pistas de subtitulos (ES, EN $C A)$, superposiciones (SDK) y bloques para saltos, preguntas y respuestas (EDL).

\subsection{Tipologías básicas de la utilización de preguntas en docencia}

Una de las técnicas habituales en la docencia para captar la atención del estudiante y mantener su interés durante la explicación es la formulación de preguntas por parte del profesor. Por lo tanto, creemos que la introducción de distintos tipos de preguntas en los videos educativos es un avance muy importante.

Así, a lo largo del mismo vídeo, se pueden utilizar varias tipologías para conseguir que el estudiante no pierda la atención y aprenda mejor los contenidos. A modo de ejemplo, se ha hecho una selección de las tipologías básicas que se podrían aplicar, pero el sistema puede configurar muchas más, con la combinación de más de una tipología, como veremos más adelante.

\subsubsection{Pausa y revisión}

Es la tipología más sencilla. Permite parar el vídeo en un punto en concreto y preguntar al estudiante si quiere continuar o prefiere volver a reproducir el vídeo desde un punto que ya ha marcado el profesor para ver de nuevo la explicación.

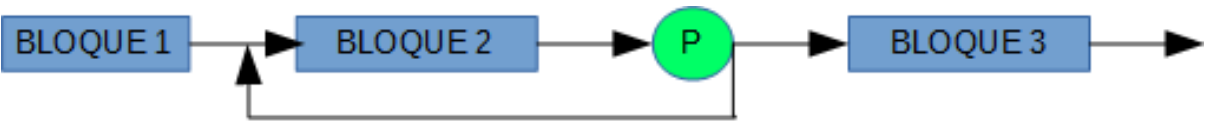

Fig. 9 Tipología 1. Pausa y revisión.

\subsubsection{Pausa para ver contenidos previos}

Se pausa el vídeo para que el estudiante pueda alcanzar los conocimientos básicos mediante un enlace a una página web u otro vídeo online, que se abrirá en una pestaña aparte del navegador, antes de continuar con el siguiente contenido. 


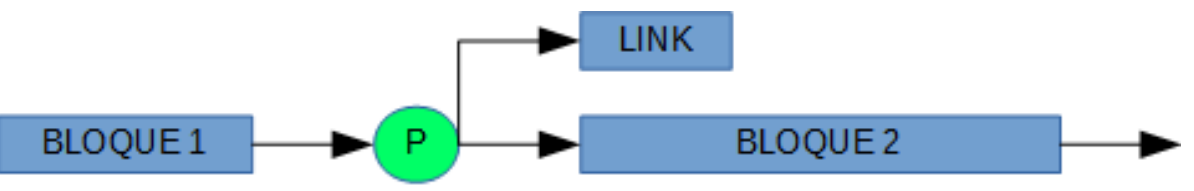

Fig. 10 Tipología 2. Pausa para ver contenidos previos.

\subsubsection{Pausa para ver contenidos extra}

Se pausa el vídeo para dar opción al estudiante a ver un contenido extra. Por ejemplo, el profesor le da la opción al estudiante de ver la demostración de una fórmula, antes de continuar con la resolución del problema donde se aplica dicha fórmula. El estudiante decide si quiere ver la demostración o seguir con la resolución del problema.

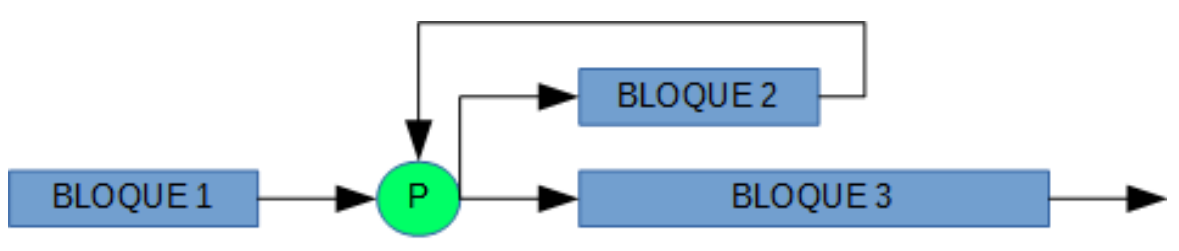

Fig. 11 Tipología 3. Pausa para ver contenidos extra.

\subsubsection{Pregunta con varias respuestas}

Se pausa el vídeo y se propone una pregunta o ejercicio con diferentes respuestas posibles, donde sólo una es correcta. El estudiante escogerá una respuesta y, si es errónea, el vídeo salta al punto de la explicación donde el profesor comenta que es errónea y por qué, volviendo de nuevo a la pregunta inicial.

También puede simplificarse visualizando siempre el mismo vídeo para todas las respuestas erróneas antes de volver a la pregunta.

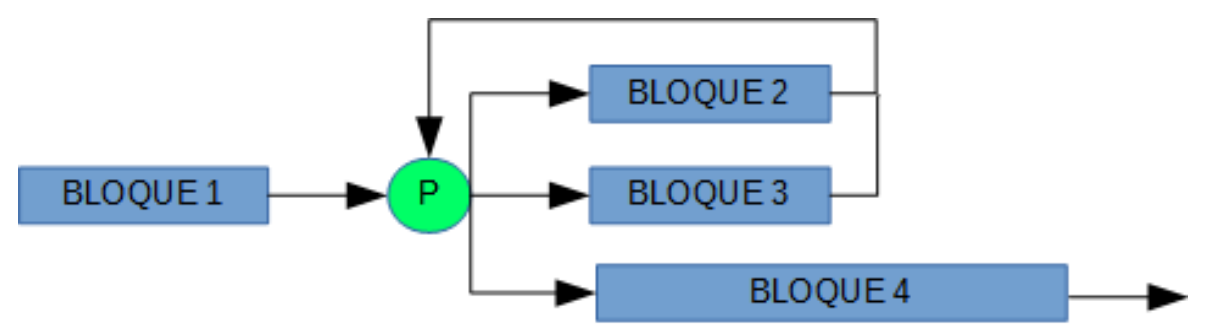

Fig. 12 Tipología 4. Pregunta con varias respuestas.

\subsubsection{Proceso en paralelo}

En un determinado punto del video, se permite al estudiante seleccionar qué camino quiere seguir para la resolución de un problema, por ejemplo, el camino largo o el corto, de forma 
gráfica o analítica, etc. De esta manera, se adapta mejor al ritmo y estilo de aprendizaje de cada uno.

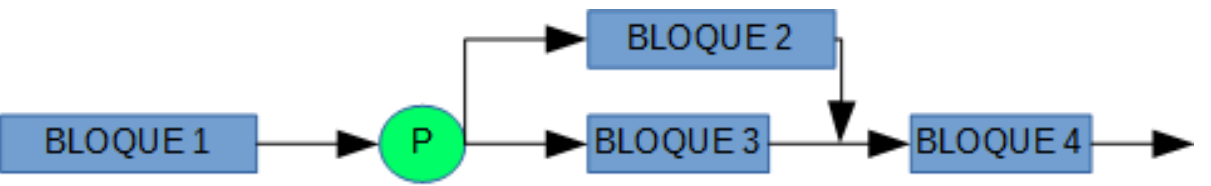

Fig. 13 Tipología 5. Proceso en paralelo.

\subsubsection{Ejemplo de implementación con varias tipologías}

Además de los casos anteriores, hay que destacar que también se puede hacer una mezcla de todas las opciones que hemos presentado en un punto como, por ejemplo, que para que el estudiante conteste a una pregunta propuesta por el profesor se le proporcione un enlace a una página web con más información u otro vídeo para la visualización, a la vez que tiene la opción de revisar el último concepto explicado si no le quedó claro.

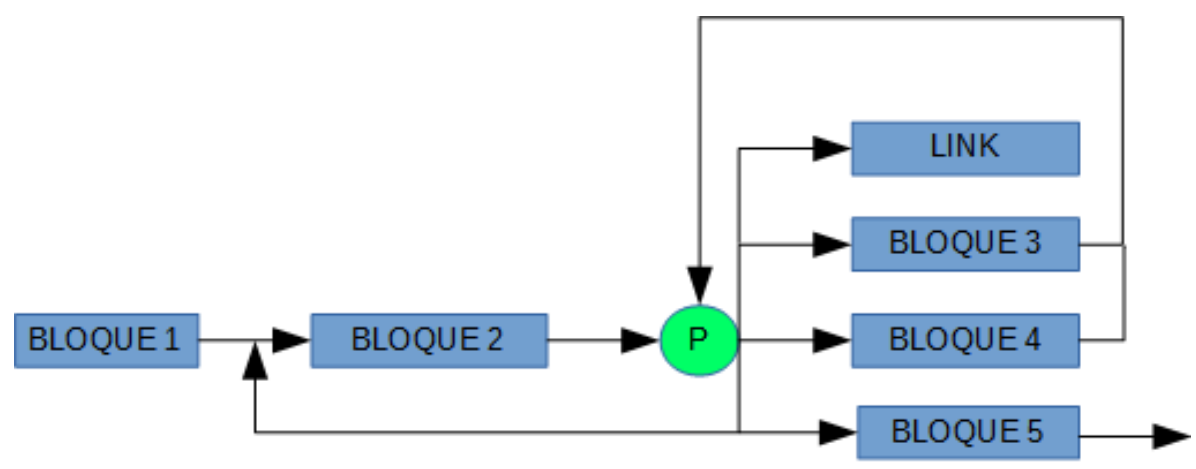

Fig. 14 Utilización de varias tipologías en una misma pregunta.

\section{Resultados}

La incorporación de todos estos elementos en los vídeos generados como materiales de apoyo a la docencia, creemos que conseguirán una mayor implicación de los estudiantes, con lo que su motivación aumentará y, con ella, y en mayor medida, su aprendizaje.

Además, se conseguirá que cada estudiante visualice el material audiovisual adaptándolo a su propio ritmo y estilo de aprendizaje, puesto que los diferentes bloques programados por el profesor así lo permitirán.

\section{Conclusiones}

La tecnología ha permitido revolucionar nuestras vidas y también nuestra forma de enseñar y aprender. En este trabajo, hemos presentado una nueva aportación del GREM de la UPV que va a facilitar a nuestro profesorado la producción de material audiovisual docente de 
mayor calidad al introducir de forma explícita interactividad con menús y botones que van a ayudar al profesor a planificar cómo tiene que seguir el estudiante la explicación correspondiente. Esperamos que, siguiendo en esta línea, el GREM de la UPV pueda, poco a poco, incorporar todos estos avances en los materiales producidos por nuestros profesores, facilitando la tarea en el menor tiempo posible.

\section{Referencias}

AMAYA, V.: La pregunta como procedimiento didáctico en el aula de ELE. Revista electrónia de didáctica, $\mathrm{n}^{\mathrm{o}}$ 5. Disponible en http://www.mecd.gob.es/dctm/redele/MaterialRedEle/Revista/2005 05/2005 redELE_5 02Anaya.pdf?documentId=0901e72b80dfa2ce

OLMEDO JARA, R. (2014). Selección, elaboración, adaptación y utilización de materiales, medios y recursos didácticos en Formación Profesional para el Empleo. SSCE0110. IC Editorial.

SALINA, J. (2002). Medios didácticos para una nueva Universidad. Jornada sobre Innovación: El aprendizaje en entornos virtuales. Universidad Pública de navarra, Pamplona 18 abril.

SOTOS SERRANO, M. Las preguntas en el aula. Análisis de la interacción educativa. Disponible en https://previa.uclm.es/ab/educacion/ensayos/pdf/revista16/16_16.pdf

UNIVERSIDAD POLITÉCNICA DE VALENCIA. Los objetos de aprendizaje como recurso para la docencia universitaria: criterios para su elaboración. http://www.upv.es/contenidos/DOCENRED/infoweb/docenred/info/U0687016.pdf [Consulta: 29 de marzo 2017] 